\title{
Contactless heart rate and respiratory measurement for sleep monitoring
}

\author{
$1^{\text {st }}$ Yingyan HOU ${ }^{1}, 2^{\text {nd }}$ Fangzheng ZHU ${ }^{2}$ \\ \{160400105@stu.hit.edu.cn'1,160400118@stu.hit.edu.cn² $\left.{ }^{2}\right\}$ \\ School of Computer Science and Technology \\ Harbin Institute of Technology \\ Weihai, China ${ }^{1,2}$
}

\begin{abstract}
Heart rate and respiration, which are physiological indicators closely related to cardiopulmonary function, are widely used in clinical diagnosis and daily monitoring. This paper proposes a heart rate measurement based on imaging photovoltaic plethysmography (iPPG) and a respiratory measurement method of sift algorithm. We mark the region of interest by dlib. Signal is obtained by color channels separation of ROI and independent component analysis. Next we filter signal with bandpass filter and extract heart rate from the blood volume pulse via Fourier transform. Meanwhile, the trajectory is decomposed into a set of component motions by calculating the feature points at the chest and performing principal component analysis. The composition of the respiration that is most suitable for the calculation is then selected based on its time spectrum. Finally, the peak of the trajectory corresponding to the respiration is determined. This method could be used for contactless sleep monitoring .
\end{abstract}

Keywords: heart rate; respiration; contactless; sleep monitoring

\section{Introduction}

In recent years, with the improvement of computer performance, video image processing technology can be used in medical diagnosis, health monitoring and other aspects to provide support for the development of modern medicine. Especially, heart rate and respiratory are widely used in clinical diagnosis and daily monitoring [1].

The traditional detection methods are mostly contact devices. The traditional heart rate measurement method is electrocardiogram (ECG) [2]. It requires the testee to stick electrodes or wear chest bands on the body, causing certain stimulation and discomfort to the testee. Photoplethysmo Graphy (PPG) is a noninvasive method to detect blood volume changes in living tissues by photoelectric means [3]. When a certain wavelength of light is irradiated on the skin surface of the fingertip, the light beam will be transmitted to the photoelectric receiver through transmission or reflection. Although compared with the traditional heart rate measurement method, the comfort is improved. It is still a contact and longterm measurement which would make the testee feel uncomfortable.

The detection methods of respiratory signals include pressure sensor method, temperature sensor method and impedance method. Pressure sensor method measure respiratory frequency by sensing the periodic deformation of respiratory duct and thorax and abdomen according to the periodic change of exhalation and inspiration. Pressure sensors are usually placed in the 
respiratory tract of adults. Children need to tie the sensors to their abdomen with straps, which will bring discomfort to the subjects. The influence of restraint pressure on respiration will bring into the measurement results, causing errors. Temperature sensor method can change the temperature in nasal cavity when gas is exchanged with external gas through nasal cavity. The physical characteristics of some materials or components are related to temperature. The change of temperature is transformed into the change of electric quantity, and the change of respiration is measured. Temperature sensor method also has the disadvantage that pressure sensor method brings discomfort to subjects.

As people pay more attention to their own health, there is a great demand for a scheme that can detect physiological indicators such as heart rate at home without going to the hospital. Therefore, the research of noncontact physiological signal measurement equipment has gradually attracted attention. The ability to monitor a patient's physiological signals by a noncontact mean has a tantalizing prospect that would enhance the delivery of primary health care.

\section{Literature review}

\subsection{Heart rate}

$\mathrm{T} \mathrm{Wu}$ et al. proposed a noncontact imaging photovoltaic plethysmography (iPPG) [4] based on the traditional photovoltaic plethysmography (PPG) technique requiring contact measurement. With the beating of the heart, the amount of blood in the blood vessels is constantly changing. When the heart pumps blood, the amount of blood in the blood vessels increases, so does the absorption of light by the blood vessels on the skin surface. And the absorption of light by other tissues remains unchanged. When the blood flows back to the heart, the amount of blood in the blood vessels decreases, and the absorption of light by the blood vessels on the skin surface decreases, while the absorption of light by other tissues also decreases. That is to say, the changes of the reflected light of skin can reflect the condition of cardiovascular activity. By processing the waveform of the reflected light of skin in a period of time, physiological signals such as heart rate can be obtained. This is the basic principle of iPPG. They irradiate skin surface with $870 \mathrm{~nm}$ infrared light as light source, receive reflected light through a black and white camera with filter, and select the same position as the ROI in each frame. Calculating the waveform of the gray mean value in ROI, we obtain the PPG signal which reflects the change of blood flow in the skin region.

Ming Zher Poh of MIT and others published a series of articles and proposed a noncontact physiological measurement scheme based on cameras [5]. They collect video signals with ordinary network cameras under natural light conditions by imaging photovoltaic plethysmography (iPPG) technology. After processing and analysis, they can get blood volume pulse wave. Further processing can get heart rate, which is low cost and high accuracy.

YuSun et al. of the National University of Singapore use iPPG technology to measure the pulse variation signal in a noncontact way [6]. They use $880 \mathrm{~nm}$ infrared light source and highspeed CMOS camera to collect video signals. After comparing the results obtained by the method and the traditional contact measurement method, they find that the results obtained by the two methods are identical.

The studies of T Wu, Yu Sun and Ming Zher Poh provide support for the measurement of some physiological parameters under noncontact conditions using simple equipment. 


\subsection{Respiratory rate}

As for the research of respiratory rate(RR) detection, the noncontact respiratory rate detection is the main research focus in the world. S. Min and others proposed a simplified structural textile capacitive respiration sensor (TCRS) for respiration monitoring system [7], which is based on capacitive pressure sensing method.

Fatih Erden and A. Enis Çetin used infrared sensors to detect Respiratory rate [8]. The system, which consists of two pyro-electric infrared (PIR) sensors, is capable of estimating the respiratory rate and detecting the sleep apnea. Mohammod Abdul Motin and others proposed an EEMD-PCA approach to extract heart rate and respiratory rate from PPG signal [9]. They used an algorithm based on ensemble empirical mode decomposition with principal component analysis (EEMD-PCA) as a novel approach to estimate HR, RR and RA simultaneously from PPG signal

Linovi and others proposed detecting respiratory rate based on EVM. Rate detection method [9]. Shourjya Sanyal and Koushik Kumar Nundy use smartphone cameras to measure heart rate (HR) and Respiratory Rate(RR) [11]. They introduced a novel iPPG method where by measuring variations in color of reflected light and can therefore measure both HR and RR from the video of a subject's face. David Lowe of University of British Columbia proposed the SIFT algorithm and improved it in 2004 [11][13]. This description has scale invariance and can detect key points in images. It is a local feature descriptor. Hao Yuwu of MIT and others proposed a method called Eulerian Video Mag nification (EVM) [14], which processes video images in both spatial and temporal domains and makes subtle changes that are not easily observed by the naked eye.

We propose a heart rate measurement based on imaging photovoltaic plethysmography (iPPG) and a respiratory measurement method of sift algorithm, which could be used for sleep monitoring. The method is first described, and then multiple sets of experiments are performed to verify its accuracy with the pulse oximeter measurement results.

\section{Heart rate measurements}

This design is divided into four parts: face detection and the mark of two regions of interest (ROI), signal separation, signal processing, and heart rate extraction from the blood volume pulse (BVP). Extracting frames from video, we mark the ROI if face is detected from frame. Signal is obtained by color channels separation of ROI and independent component analysis. We smooth the signal by detrending and demeaning and filter signal with bandpass filter. At last, we extract heart rate from the BVP via Fourier transform.

\subsection{Location of ROI}

We utilized dlib to detect faces within the video frames and mark two regions of interest [15]. Dlib implements the algorithm described in the paper One Millisecond Face Alignment with an Ensemble of Regression Trees, by Vahid Kazemi and Josephine Sullivan [16]. Using opencv for face detection, some faces can not be detected, and accuracy is low when detecting the side face. Compared with opencv, dlib is more accurate, and it could detect 68 feature 
points or even more on the face. We locate the ROI by the position of the feature points, as shown in the Figure 1.

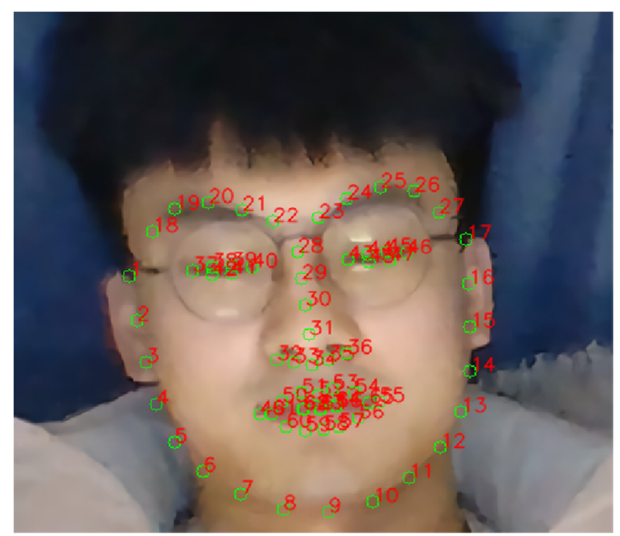

Fig.1. The position of the feature points

The feature extractor (predictor) requires a rough bounding box as input to the algorithm. This is provided by a traditional face detector. Detector returns a list of rectangles, each of which corresponds with a face in the image. In the method of Ming Zher Poh [5], they selected the center $60 \%$ width and full height of the box as the ROI for subsequent calculations. However, there would be some interference due to the movement of the eyes. Compared with marking region of full face, combination of forehead and nose performs better. For each face detected, we localize the region of interest. Nose, partial cheeks and forehead area between eyebrows are selected as ROI. We draw two rectangular frames and extract the ROI as the signal extraction area, as shown in the Figure 2.

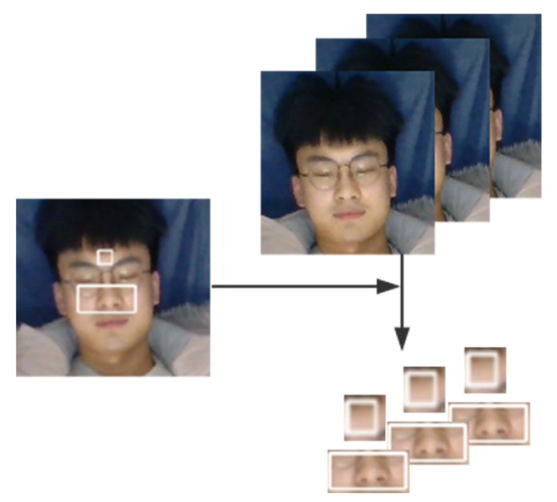

Fig.2. Extract ROI from frame

Although reducing the area of the ROI may lead to an increase of noise, the regions we selected are in the high-frequency regions, which are easy to filter out and have less influence on measuring heart rate.

\subsection{Signal separation}


The regions of interest are used as a signal extraction area, which is separated into three RGB channel signals of red, green and blue. We split three primary colors for two regions of interest per frame, and select RGB signal formats. The nose (ROI1) area size is $R \times S$, and the forehead (ROI2) area size is $P \times Q$. The mean $\bar{x}_{k}$ of every channel in ROI1 and the mean $\bar{y}_{k}$ of every channel in ROI2 is calculated as follows equations (1) and (2):

$$
\begin{gathered}
\bar{x}_{k}=\frac{\sum_{i=1}^{R} \sum_{j=1}^{S} x_{k}}{R \times S} \quad(k=1,2,3) \\
\bar{y}_{k}=\frac{\sum_{i=1}^{P} \sum_{j=1}^{Q} y_{k}}{P \times Q} \quad(k=1,2,3)
\end{gathered}
$$

Get the signals of the three channels of RGB: $\bar{x}_{1}(t), \bar{x}_{2}(t), \bar{x}_{3}(t)$ and $\bar{y}_{1}(t), \bar{y}_{2}(t), \bar{y}_{3}(t)$, where $t$ is the time series of the video frames. Calculate the average intensity, see equation(3):

$$
r_{k}(t)=\left(\bar{x}_{k}(t)+\bar{y}_{k}(t)\right) / 2 \quad(k=1,2,3)
$$

Next, we use the blind signal separation to separate the RGB three color channel and find the frequency from three independent components. Blind signal separation (BSS) is the separation of a set of source signals from a set of mixed signals, without the aid of information about the source signals or the mixing process. Independent component analysis (ICA), a special case of blind source separation, is a computational method for separating a multivariate signal into additive subcomponents [17]. In this experiment, we assume that there are three independent source signals: $s(t)=\left[s_{1}(t), s_{2}(t), s_{3}(t)\right]^{T}$, and the observed signals are three color channel signals $r(t)=\left[r_{1}(t), r_{2}(t), r_{3}(t)\right]^{T}$. The linear mixed model of ICA can be expressed as follows equation (4):

$$
r(t)=A s(t)
$$

where $A \in R^{3 \times 3}$ is an unknown mixed matrix. Three source signals $s(t)$ are mixed to obtain the observed signals $r(t)$. Determining the separation matrix $W$ based on $r(t)$ as follows equation (5):

$$
v(t)=A^{-1} r(t)=W r(t)
$$

where $v(t)=\left[v_{1}(t), v_{2}(t), v_{3}(t)\right]^{T}$ is an estimate of the $s(t)$. FastICA is an efficient algorithm for independent component analysis. The specific steps are as follows:

(1) Prewhiten and center the data, and get $Z \leftarrow r(t)$

(2) Randomize the initial weight vector $W$

(3) Iterative update: $w_{i} \leftarrow E\left\{Z g\left(w_{i}^{T} Z\right)\right\}-E\left\{g^{\prime}\left(w_{i}^{T} Z\right)\right\} w$, where $E\{\ldots\}$ means averaging over all column vectors of matrix $Z$. And let $W \leftarrow\left(W W^{T}\right)^{-1 / 2} W$.

(4) If not converged, go back to (3)

It is considered that the $\mathrm{G}$ channel almost always features a much stronger HR signal as compared to the $\mathrm{R}$ and $\mathrm{B}$ channels. And it is also a strong evidence that the signals are filtered 
by variations in blood volume (due to the absorption bands for oxy and deoxy hemoglobin for yellow and green light) [18][19]. Correlation between the three independent signals $v(t)$ and the green channel signal $r_{2}(t)$ was analyzed to obtain the signal with the best correlation. The calculation results show that the best correlation with the green channel is the component $v_{2}(t)$.

Based on this, we studied the effect of noise on the color channel. Heart rate and noise resistance were tested with individual color channels. Each color channel was used for contrast test with 15 videos of the same frame rate. Next, we performed the noise comparison test by adding gaussian noise to videos, and calculated the average error. Record the test results, as shown in Table 1.

Table 1. The number of wave crest

\begin{tabular}{ccc}
\hline Color channel & $\begin{array}{c}\text { The mean error } \\
\text { (no noise) }\end{array}$ & $\begin{array}{c}\text { The mean error } \\
\text { (add noise) }\end{array}$ \\
\hline R & 3.05 & 3.54 \\
G & 0.26 & 1.23 \\
B & 3.57 & 4.31 \\
RGB & 2.44 & 3.07 \\
\hline
\end{tabular}

From the test, the green channel does have a better performance for heart rate detection, while also reducing noise.

\subsection{Signal processing}

We consider the $v_{2}(t)$ as a continuous function of time, and filter the signal with a bandpass filter for the time series of $v_{2}(t)$. In this paper, we extract the heart rate signal, so the time domain filtering uses a bandpass filter of $0.83-3.33 \mathrm{~Hz}$ (heart rate $50-200$ times $/ \mathrm{min}$ ).

We plot the mean value $\bar{v}_{2}(t)$ as a function of time, which is the blood volume pulse(BVP), as shown in the Figure 3.

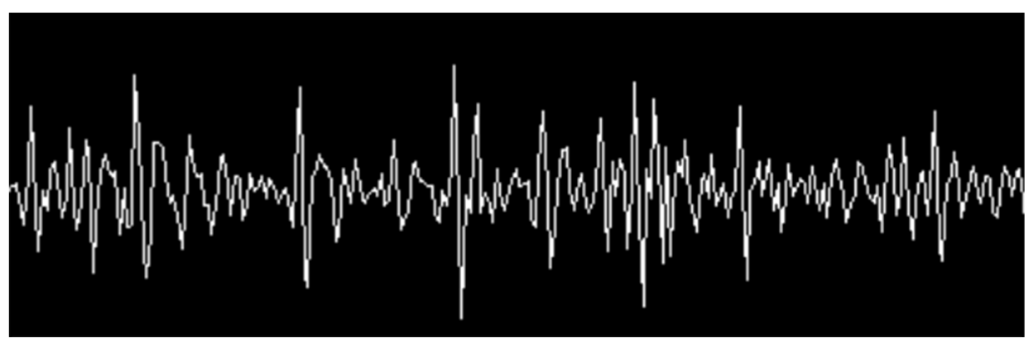

Fig.3. The blood volume pulse

\subsection{Heart rate extraction from BVP}


The BVP signal is converted into a frequency domain signal via fourier transformed. Analytical power spectrum, and the peak of the power spectrum is the heart rate $f$. The heart rate could be calculated, as follows equation (6):

$$
H R=f \times 60
$$

Since the heart rate change between samples does not exceed $10 \%$, a weighted average is used to smooth the BPM with the last BPM, see equation(7):

$$
x 1=x 2 \times 0.9+x 1 \times 0.1
$$

where $x 1$ means the BPM, and $x 2$ means the last BPM. Taking bpm as the abscissa and power as the ordinate, the abscissa value corresponding to the power peak is 65 times $/ \mathrm{min}$, as shown in the Figure 4.

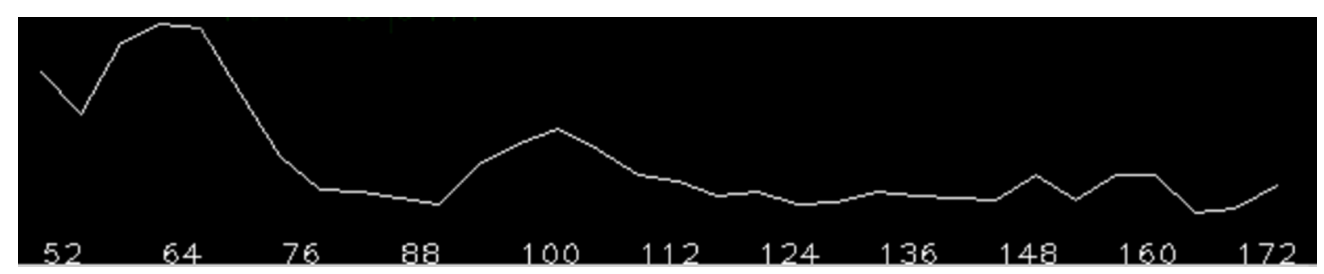

Fig.4. The power spectrum of BVP

\section{Respiratory rate measurements}

The detection of respiratory frequency consists of five parts. First, we use Eulerian Video Magnification to amplify the subtle movement we interested. Then, using sift algorithm to find feature points. Next step is Temporal Filtering. Finally, we use PCA to decompose this mixed motion and finish peak detection.

\subsection{Use Eulerian Video Magnification to amplify movement}

Sometimes respiration movements is very subtle, we need to use Eulerian Video Magnification to amplify the movement we interested. Hao Yuwu of MIT and others proposed a method called Eulerian Video Mag nification (EVM) [14], which processes video images in both spatial and temporal domains and makes subtle changes that are not easily observed by the naked eye.

Assuming that the whole image is constantly changing, but these signals have different amplitude and frequency characteristics. The signal of change is hidden in it. In this way, the magnification of these weak changes which is essentially to select and enhance the frequency bands to be studied. In 2012, Wu et al. proposed a method called Eulerian Video Magnification from this perspective. The process is as follows:

1.Spatial filtering. Pyramid multi-resolution decomposition of video sequence is performed.The generation of the Gauss pyramid can be expressed as follows equation (8):

$$
\operatorname{GaussI}_{i}=\left[\omega * \operatorname{GaussI}_{i}+1\right] \downarrow
$$


where GaussI ${ }_{i}$ is layer $i$ of the Gauss Pyramid, $\downarrow$ means down sampling, $\star$ as convolution operator, $\omega$ as gaussian kernel.Laplacian pyramid is obtained by the difference between two layers of images in the Gauss pyramid. If $i$ represent Laplacian Pyramid's Layer I, then see equation (9):

$$
\text { Lapli }=\text { GaussI }_{i}-\operatorname{Pyr} U p\left(\text { GaussI }_{i}+1\right)
$$

where Lapli is the Up-sampling of Gauss pyramid, Gauss $I_{i}$ is layer $i$ of the Gauss Pyramid, PyrUp is layer I of the Laplacian Pyramid.

2.Time domain filtering. Time domain bandpass filtering is applied to each scale image to obtain several frequency bands of interest.

3.Amplify the filtering results. For each frequency band, the results of differential approximation and linear amplification approximation are obtained by Taylor series.

4.Composite image. Synthesize the enlarged image.

\subsection{Tracking and find feature points}

We use sift algorithm from OPENCV 3.4 to find the feature points. Lowe proposed a method called Scale-invariant feature transform [12][13], Scale-invariant feature transform is a computer vision algorithm used to detect and describe local features in images.

Lowe decomposes the SIFT algorithm into the following four steps:

1. Scale Space Extremum Detection: Search for image positions on all scales. Gauss differential function is used to identify potential points of interest for scale and rotation invariance.

2. Key Point Location: At each candidate position, the position and scale are determined by a well-fitted model. The selection of key points depends on their stability.

3. Direction determination: Based on the local gradient direction of the image, one or more directions are assigned to each key point position. All subsequent operations on image data are transformed relative to the direction, scale and location of key points, thus providing invariance for these transformations.

4. Key Point Description: In the neighborhood around each key point, the local gradient of the image is measured on the selected scale. These gradients are transformed into a representation that allows for larger local shape deformations and light changes.

Through the above steps, SIFT feature point location, scale and direction information has been found. Next, we need to use a set of vectors to describe the key point, which is also to generate feature point descriptors. This descriptor contains not only feature points, but also the pixels around the feature points that contribute to it. The descriptor should be independent to ensure the matching rate.

In order to enhance the robustness of matching, it is proposed to use $4 \times 4$ seed points to describe each key point. Such a key point can generate 128-dimensional SIFT feature vectors.

We then use BFMatcher method to find the best match and only take the first 10 points with the greatest change in the axis of the best matching as the feature points of the calculation, as shown in the Figure 5. Between frame 1 and each frame $\mathrm{t}=2$ to $\mathrm{T}$, we get the location $\left\{x_{\mathrm{n}}(t), y_{\mathrm{n}}(t)\right\}$ for each feature point $\mathrm{n}$. And we only analyze the vertical component $y_{\mathrm{n}}$. 


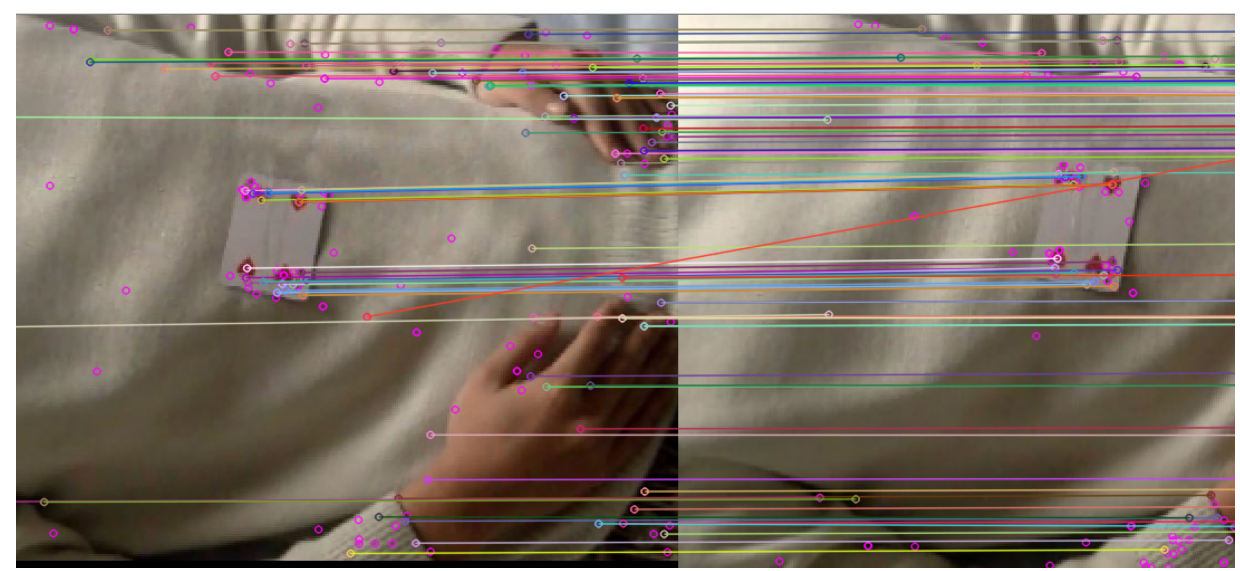

Fig.5. Use sift to do feature points matching

\subsection{Temporal Filtering}

Not all frequencies of the trajectories are required or useful for respiratory rate detection. A normal baby's respiratory rate falls within $[0.25,2] \mathrm{Hz}$, or $[15,60]$ times/min. On the one hand, the movements' frequencies lower than $0.25 \mathrm{~Hz}$ usually like changes in posture, which negatively affect our respiratory rate detection. On the other hand, the frequencies higher than $2 \mathrm{~Hz}$ provide useful precision needed for peak detection. Taking these elements into consideration, we filter each feature point vertical component $y_{n}$ to a passband of $[0.25,5] \mathrm{Hz}$. We use a 5th order Butterworth filter.

\subsection{Use PCA to decompose this mixed motion}

The only source signal we're interested in is the movement of belly caused by baby's respiration. Therefore, we need to decompose this mixed motion to isolate the respiratory signal. To do this we consider the multidimensional position of the belly at each frame as a separate data point and use PCA [20] to extract the main linear components of data by transforming the original data into a set of linear independent representations. We then select a dimension on which to project the position time series to get the respiratory signal. If there are $\mathrm{X}$ feature points, the Ndimensional position of the feature points at frame $t$ as $n_{t}=$ $\left[y_{1}(t), y_{2}(t), \cdots, y_{n}(t)\right]$. we can get the covariance matrix:

$$
\sum_{\mathrm{n}}=\sum_{i=1}^{T}\left(n_{t}-\bar{n}\right)\left(n_{t}-\bar{n}\right)^{T}
$$

where $\sum_{\mathrm{n}}$ is the covariance matrix, $\bar{n}$ is the mean of samples, $T$ is the number of frames. And we can use PCA to put the principal axes of variation of the position as the eigenvectors of the covariance matrix:

$$
\sum_{\mathrm{n}} \Phi_{n}=\Phi_{n} R_{n}
$$


where $R_{n}$ is a diagonal matrix contains the eigenvalues $\lambda_{1}, \lambda_{2}, \cdots, \lambda_{X}$ corresponding to the eigenvectors in the columns of $\Phi_{n}$. The N-dimensional direction and motion amplitude of each feature point are shown by eigenvectors.

\subsection{Peak detection and calculate respiratory rate}

We will choose the most periodic eigenvectors and do fourier transform. We then do the peak detection. We perform peak detection on the selected PCA component signal. The peaks are close to $1 /$ frate seconds apart with some variability due to the natural variability of respiration, variations of the belly motion, and noise. We label each sample in the signal as a peak if it is the largest value in a window centered at the sample and use peak value. And the respiratory rate is calculated by peak value via fourier transform.

\section{Experimental work}

\subsection{Experimental setup}

To verify the accuracy of the heart rate and respiration methods, we enrolled 5 healthy male college students and 5 healthy female college students to experimentize. We measured the reference value of HR and RR using ECG device (HR) and self-reporting (RR). Measure 5 minutes for each volunteer, and record the heart rate and respiration every 20 seconds. A total of 150 sets of data were obtained. The volunteers were lying on their back.

\subsection{Work method}

The differences between the measured value and the reference value was clarified by using Bland-Altman analysis. The abscissa indicates the average of the two measurement results in the same experiment, and the ordinate indicates the difference between the two methods in the experiment. The middle solid line indicates the mean $\bar{d}$ of the difference between the multiple experiments, and the two broken lines indicate the upper and lower limits of the $95 \%$ limits of agreement $\bar{d} \pm 1.96 S D$, where $S D$ is the standard deviation of 150 experimental difference values. The higher degree of agreement between the two measurement methods is, the closer the solid line representing the difference mean is to 0 , and the less data would be outside the limits of agreement.

Using the heart rate method of this paper, $\bar{d}$ was -0.2913 with $95 \%$ limits of agreement 2.7502 to 2.1675 , reflecting heart rate consistency. $94.00 \%$ (141/150) of the points are within the $95 \%$ limits of agreement, as shown in the Figure 6(a). Using the respiration method of this paper, $\bar{d}$ was -0.5113 with $95 \%$ limits of agreement -3.6901 to 2.6674 , reflecting respiration consistency. $98.67 \%(148 / 150)$ of the points are within the $95 \%$ limits of agreement, as shown in the Figure 6(b). It can be seen that the heart rate and respiration methods in this paper have a high consistency with the traditional method. 


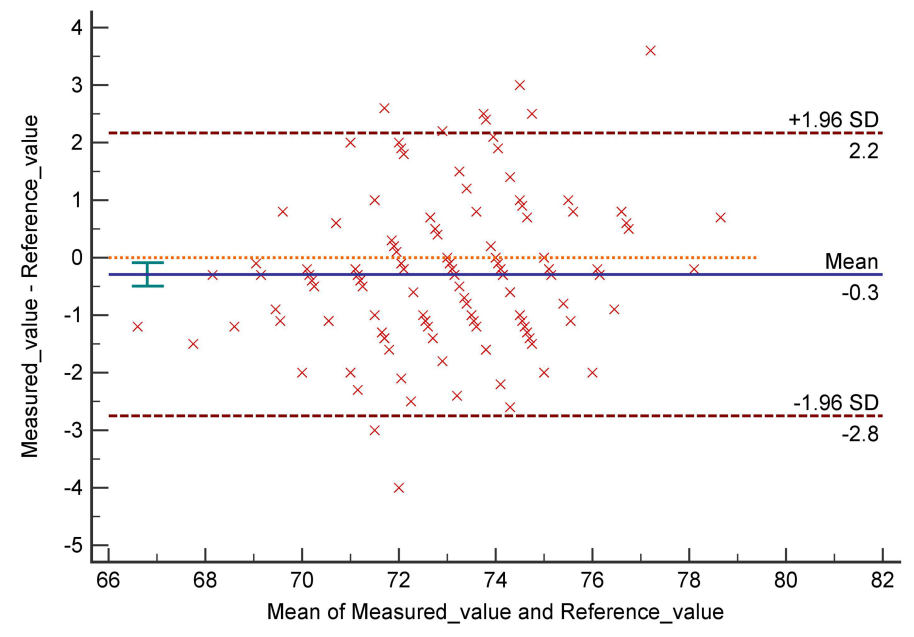

(a) the accuracy of the heart rate by Bland-Altman analysis

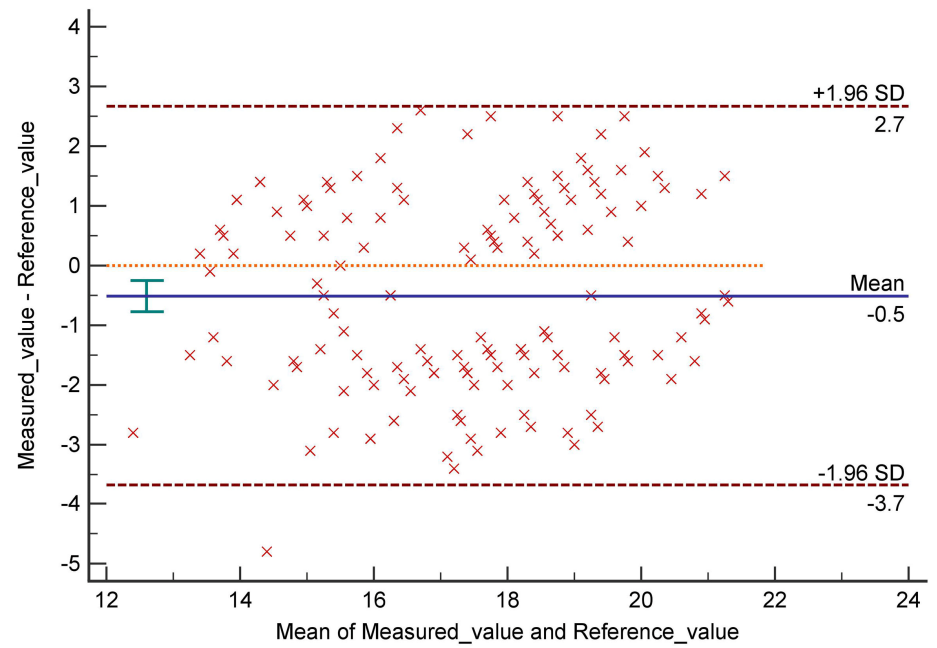

(b) the accuracy of the respiration by Bland-Altman analysis

Fig.6. The accuracy of method by Bland-Altman analysis

\section{Conclusion}

In this paper, we realize that measure heart rate and respiration using the common camera without contact by recognizing facial feature points and abdominal feature points. It has been proved by experiments that the method could get comparatively accurate results. 
Considering those characteristics of simplicity and convenience, the method in this paper is suitable for daily sleep monitoring, especially for the old men and infants whom need special care, and could be well applied. In addition, it also has a good application prospect in the detection of lies and other aspects. At the same time, we would study the detection of heart rate and respiration by infrared light under dark conditions, which is the direction of our future efforts.

\section{Acknowledgments}

We wish to acknowledge Professor Shengping Zhang, Harbin Institute of Technology, for his guidance and help in this study. Without his insightful and patient guidance, we cannot successfully complete this paper.

\section{References}

[1] Kannel, William B., et al.: Heart rate and cardiovascular mortality: the Framingham Study. American heart journal 113.6, pp. 1489-1494 (1987)

[2] Zijlmans, M D. Flanagan ,and J. Gotman.: Heart rate changes and ECG abnormalities during epileptic seizures: prevalence and definition of an objective clinical sign. Epilepsia 43.8, pp. 847-854 (2010)

[3] Allen, John.: Photoplethysmography and its application in clinical physiological measurement. Physiological Measurement 28.3,:R1 (2007)

[4] Wu, Ting, Vladimir Blazek, and Hans Juergen Schmitt.: Photoplethysmography imaging: a new noninvasive and noncontact method for mapping of the dermal perfusion changes. Optical Techniques and Instrumentation for the Measurement of Blood Composition, Structure, and Dynamics. Vol. 4163. International Society for Optics and Photonics (2000)

[5] Poh, Ming-Zher, Daniel J. McDuff, and Rosalind W. Picard.: Non-contact, automated cardiac pulse measurements using video imaging and blind source separation. Optics express 18.10, pp. 10762-10774 (2010)

[6] Sun, Yu, et al.: Noncontact imaging photoplethysmography to effectively access pulse rate variability. Journal of biomedical optics 18.6: 061205 (2012)

[7] Min, Se Dong, Yonghyeon Yun, and Hangsik Shin. Simplified structural textile respiration sensor based on capacitive pressure sensing method. IEEE Sensors Journal 14.9, pp. 3245-3251 (2014)

[8] Fatih Erden, A.: Enis Çetin Respiratory rate monitoring using infrared sensors Electrical and Electronics Engineering (ELECO), 2015 9th International Conference on. IEEE, pp. 1136-1140 (2015)

[9] Mohammod Abdul Motin, Chandan Kumar Karmakar, Marimuthu Palaniswami.: An EEMDPCA approach to extract heart rate, respiratory rate and respiratory activity from PPG signal. Engineering in Medicine and Biology Society (EMBC), 2016 IEEE 38th Annual International Conference of the. IEEE. pp. 3817-3820 (2016)

[10] Alinovi, Davide, et al.: Spatio-temporal video processing for respiratory rate estimation. Medical Measurements and Applications (MeMeA), 2015 IEEE International Symposium on. IEEE. pp. 12-17 (2015)

[11] Shourjya Sanyal, Koushik Kumar Nundy.: Algorithms for Monitoring Heart Rate and Respiratory Rate From the Video of a User's Face. IEEE Journal of Translational Engineering in Health and Medicine Volume. pp. 1-11 (2018)

[12] Lowe, David G.: Distinctive image features from scale-invariant keypoints. International journal of computer vision 60.2, pp. 91-110 (2004)

[13] Lowe, David G.: Object recognition from local scale-invariant features. Computer vision, 1999. The proceedings of the seventh IEEE international conference on. Vol. 2, pp. 1150-1157 (1999) 
[14] $\mathrm{Wu}$, Hao-Yu, et al.: Eulerian video magnification for revealing subtle changes in the world. (2012)

[15] King, Davis E.: Dlib-ml: A machine learning toolkit. Journal of Machine Learning Research. pp. $1755-1758$ (2009)

[16] Kazemi, Vahid, and Josephine Sullivan.: One millisecond face alignment with an ensemble of regression trees. Proceedings of the IEEE Conference on Computer Vision and Pattern Recognition. pp. 1867-1874 (2014)

[17] Lee, Te-Won.: Independent component analysis. Independent component analysis. Springer, Boston, MA. pp. 27-66 (1998)

[18] Zheng,Jia, et al.: Feasibility of imaging photoplethysmography. BioMedical Engineering and Informatics, 2008. BMEI 2008. International Conference on IEEE. Vol. 2, pp. $72-75$ (2008)

[19] Verkruysse, Wim, Lars O. Svaasand, and J. Stuart Nelson.: Remote plethysmographic imaging using ambient light. Optics express 16.26: 21434-21445 (2008)

[20] Wold, Svante, Kim Esbensen, and Paul Geladi.: Principal component analysis. Chemometrics and intelligent laboratory systems. pp. 37-52 (1987) 